Article

\title{
Verifying the Firoozbakht, Nicholson, and Farhadian Conjectures up to the 81st Maximal Prime Gap
}

\author{
Matt Visser ${ }^{\mathbb{D}}$ \\ School of Mathematics and Statistics, Victoria University of Wellington, P.O. Box 600, \\ Wellington 6140, New Zealand; matt.visser@sms.vuw.ac.nz
}

Received: 9 May 2019; Accepted: 28 July 2019; Published: 30 July 2019

\begin{abstract}
The Firoozbakht, Nicholson, and Farhadian conjectures can be phrased in terms of increasingly powerful conjectured bounds on the prime gaps $g_{n}:=p_{n+1}-p_{n}$. While a general proof of any of these conjectures is far out of reach, I shall show that all three of these conjectures are unconditionally and explicitly verified for all primes below the as yet unknown location of the 81st maximal prime gap, certainly for all primes $p<2^{64}$. For the Firoozbakht conjecture itself this is a rather minor improvement on currently known results, but for the somewhat stronger Nicholson and Farhadian conjectures this may be considerably more interesting. SEQUENCES: A005250 A002386 A005669 A000101 A107578 A246777 A246776.
\end{abstract}

Keywords: primes; prime gaps; Firoozbakht conjecture; Nicholson conjecture; Farhadian conjecture MSC: 11A41 (Primes); 11N05 (Distribution of primes)

\section{Introduction}

The Firoozbakht, Nicholson, and Farhadian conjectures would, if proved to be true, impose increasingly strong constraints on the distribution of the primes; this distribution being a fascinating topic that continues to provide many subtle and significant open questions [1-23]. The Firoozbakht conjecture [24-28] is normally phrased as follows.

Conjecture 1. (Firoozbakht conjecture, two most common versions)

$$
\left(p_{n+1}\right)^{\frac{1}{n+1}}<\left(p_{n}\right)^{\frac{1}{n}} ; \quad \text { equivalently } \quad \frac{\ln p_{n+1}}{n+1}<\frac{\ln p_{n}}{n} ; \quad(n \geq 1) .
$$

To see why this conjecture might be somewhat plausible, one may use the standard inequalities $n \ln n<p_{n}<n \ln p_{n}$, which hold for $n \geq 1$ and $n \geq 4$ respectively, and observe that

$$
\frac{\ln (n \ln n)}{n}<\frac{\ln p_{n}}{n}<\frac{\ln ^{2} p_{n}}{p_{n}} ; \quad(n \geq 1 ; n \geq 4) .
$$

Now $\frac{\ln (n \ln n)}{n}$ is monotone decreasing for $n \geq 5$, and $\frac{\ln ^{2} p_{n}}{p_{n}}$ is monotone decreasing for $p_{n}>7$. So for $n \geq 5$, corresponding to $p_{n} \geq 11$, the function $\frac{\ln p_{n}}{n}$ is certainly bounded between two monotone decreasing functions; the overall trend is monotone decreasing. The stronger conjecture that $\frac{\ln p_{n}}{n}$ is itself monotone decreasing depends on fluctuations in the distribution of the primes $p_{n}$; fluctuations which can be rephrased in terms of the prime gaps $g_{n}:=p_{n+1}-p_{n}$. 
Indeed, Kourbatov [26] using results on first occurrence prime gaps [29] has recently verified Firoozbakht's conjecture to hold for all primes $p<4 \times 10^{18}$. Furthermore Kourbatov [27] has also derived a sufficient condition for the Firoozbakht conjecture to hold:

$$
g_{n}<\ln ^{2} p_{n}-\ln p_{n}-1.17 ; \quad\left(n \geq 10 ; p_{n} \geq 29\right) .
$$

Using tables of first occurrence prime gaps [29-31] and maximal prime gaps [32-40] Kourbatov has now extended this discussion [28], and subsequently verified that Firoozbakht's conjecture holds for all primes $p<1 \times 10^{19}$. More recently (2018), two additional prime gaps (known since 2014 [29]) have been proved to be maximal [32,33], yielding the 79th and 80th maximal prime gaps, so that Kourbatov's arguments can be extended to now certainly verify the Firoozbakht conjecture up to the 80th maximal prime gap-more precisely, for all primes below currently unknown location of the 81st maximal prime gap-though we do now know (September 2018) that $p_{81}^{*}>2^{64}$ [29], see also the discussion in [28]. So certainly, the Firoozbakht conjecture holds for all primes $p<2^{64}=18,446,744,073,709,551,616 \approx$ $1.844 \times 10^{19}$. Note that this automatically verifies a strong form of Cramér's conjecture

$$
g_{n}<\ln ^{2} p_{n} ; \quad\left(n \geq 5 ; p_{n} \geq 11\right),
$$

at least for all primes $p<2^{64} \approx 1.844 \times 10^{19}$.

What is tricky with Kourbatov's techniques is to say anything useful about the somewhat stronger Nicholson [41] and Farhadian [42,43] conjectures, and it is this issue we shall address below.

\section{Firoozbakht, Nicholson, and Farhadian}

When comparing the Firoozbakht conjecture with the somewhat stronger Nicholson and Farhadian conjectures it is useful to work with the ratio of successive primes, $p_{n+1} / p_{n}$.

Conjecture 2. (Firoozbakht/Nicholson/Farhadian conjectures; successive primes version)

$$
\begin{aligned}
& \left(p_{n+1} / p_{n}\right)^{n}<p_{n} \quad(n \geq 1 \text {; Firoozbakht }) . \\
& \left(p_{n+1} / p_{n}\right)^{n}<n \ln n \quad(n>4 ; \text { Nicholson }) . \\
& \left(p_{n+1} / p_{n}\right)^{n}<p_{n} \frac{\ln n}{\ln p_{n}} \quad \quad(n>4 \text {; Farhadian }) .
\end{aligned}
$$

When phrased in this way, the standard inequalities $n \ln n<p_{n}<n \ln p_{n}$ show that Farhadian $\Longrightarrow$ Nicholson $\Longrightarrow$ Firoozbakht. To study the numerical evidence in favour of these conjectures it is useful to convert them into statements about the prime gaps $g_{n}:=p_{n+1}-p_{n}$.

Conjecture 3. (Firoozbakht/Nicholson/Farhadian conjectures; prime gap version)

$$
\begin{array}{ll}
g_{n}<p_{n}\left(p_{n}^{1 / n}-1\right) & (n \geq 1 ; \text { Firoozbakht }) . \\
g_{n}<p_{n}\left((n \ln n)^{1 / n}-1\right) & (n>4 ; \text { Nicholson }) . \\
g_{n}<p_{n}\left(\left(p_{n} \frac{\ln n}{\ln p_{n}}\right)^{1 / n}-1\right) & (n>4 ; \text { Farhadian }) .
\end{array}
$$


This can further be rephrased as:

$$
\begin{array}{ll}
g_{n}<p_{n}\left(\exp \left(\frac{\ln p_{n}}{n}\right)-1\right) & (n \geq 1 ; \text { Firoozbakht }) . \\
g_{n}<p_{n}\left(\exp \left(\frac{\ln (n \ln n)}{n}\right)-1\right) & (n>4 ; \text { Nicholson }) . \\
g_{n}<p_{n}\left(\exp \left(\frac{1}{n} \ln \left(p_{n} \frac{\ln n}{\ln p_{n}}\right)\right)-1\right) & (n>4 ; \text { Farhadian }) .
\end{array}
$$

These inequalities are all of the form $g_{n}<f\left(p_{n}, n\right)$, with $f\left(p_{n}, n\right)$ a function of both $p_{n}$ and $n$.

While $p_{n}$ and $n$ are both monotone increasing, unfortunately without further conditions being imposed the functions $f\left(p_{n}, n\right)$ are not guaranteed to be monotone increasing as functions of $n$, so to numerically verify these conjectures one would in principle have to check each individual value of $n$ independently. So our strategy will be to seek to find suitable sufficient conditions for the Firoozbakht/Nicholson/Farhadian conjectures of the form $g_{n}<f(n)$, with the function $f(n)$ being some monotone function of its argument. Once this has been achieved we can develop an argument using maximal prime gaps.

\section{Sufficient Condition for the Nicholson and Firoozbakht Conjectures}

Using the fact that $e^{x}-1>x$ we deduce a sufficient condition for the Nicholson conjecture (which is then automatically also sufficient for the Firoozbakht conjecture).

Sufficient condition 1. (Nicholson/Firoozbakht)

$$
g_{n}<\frac{p_{n} \ln (n \ln n)}{n} ; \quad(n>4 ; n \geq 1)
$$

Now use Dusart's result [14] that for $n \geq 2$ we have $p_{n}>n(\ln (n \ln n)-1)$ to deduce the stronger sufficient condition

Sufficient condition 2. (Nicholson/Firoozbakht)

$$
g_{n}<f(n)=(\ln (n \ln n)-1) \ln (n \ln n) ; \quad(n>4 ; n \geq 2) .
$$

This function $f(n)$ is clearly monotone and a posteriori we shall verify that this last condition is strong enough to be useful, and weak enough to be true over the domain of interest.

\section{Verifying the Firoozbakht and Nicholson Conjectures for All Primes $p<2^{64}$}

This is a variant of the argument given for the Andrica conjecture in references [22,23]. Consider the maximal prime gaps: Following a minor modification of the notation of references [22,23], let the quartet $\left(i, g_{i}^{*}, p_{i}^{*}, n_{i}^{*}\right)$ denote the $i$ th maximal prime gap; of width $g_{i}^{*}$, starting at the $n_{i}^{*}$ th prime $p_{i}^{*}=p_{n_{i}^{*}}$. (See see the sequences A005250, A002386, A005669, A000101, A107578.)

As of April 2019, some 80 such maximal prime gaps are known [32-40], up to $g_{80}^{*}=1550$ and

$$
p_{80}^{*}=18,361,375,334,787,046,697>1.836 \times 10^{19},
$$

which occurs at

$$
n_{80}^{*}=423,731,791,997,205,041 \approx 423 \times 10^{15} .
$$

The values of $n_{i}^{*}$, for $i \leq 80$, have been verified using the program primecount [44] which relies on the Deleglise-Rivat method [45] for computing $\pi(x)$. The primecount computation took less than 4 min for each value of $n_{i}^{*}$. (For more background on prime counting, see references $[46,47]$.) 
One now considers the interval $\left[p_{i}^{*}, p_{i+1}^{*}-1\right]$, from the lower end of the $i$-th maximal prime gap to just below the beginning of the $(i+1)^{t h}$ maximal prime gap. Then everywhere in this interval

$$
\forall p_{n} \in\left[p_{i}^{*}, p_{i+1}^{*}-1\right] \quad g_{n} \leq g_{i}^{*} ; \quad f\left(n_{i}^{*}\right) \leq f(n) .
$$

Therefore, if the sufficient condition for the Nicholson/Firoozbakht conjectures holds at the beginning of the interval $p_{n} \in\left[p_{i}^{*}, p_{i+1}^{*}-1\right]$, then it certainly holds on the entire interval. (Note that for the Nicholson/Firoozbakht conjectures, in addition to knowing the $p_{i}^{*}$, it is also essential to know all the $n_{i}^{*}=\pi\left(p_{i}^{*}\right)$ in order for this particular verification procedure to work; in contrast for the Andrica conjecture one can quietly discard the $n_{i}^{*}=\pi\left(p_{i}^{*}\right)$ and only work with the $p_{i}^{*}[22,23]$.)

Explicitly checking a table of maximal prime gaps [32-40], we inspect the ratios

$$
\frac{g_{i}^{*}}{\left(\ln \left(n_{i}^{*} \ln n_{i}^{*}\right)-1\right) \ln \left(n_{i}^{*} \ln n_{i}^{*}\right)}
$$

and verify that they are less than unity for $i \in[5,80]$. Consequently both of the Nicholson and Firoozbakht conjectures certainly hold on the interval $\left[p_{5}^{*}, p_{81}^{*}-1\right]$, that is from $p_{5}^{*}=89$ up to just before the beginning of the 81st maximal prime gap, $p_{81}^{*}-1$, even if we do not yet know the value of $p_{81}^{*}$. Then explicitly checking the primes below $p_{5}^{*}=89$ the Firoozbakht conjecture holds for all primes $p$ less than $p_{81}^{*}$, while the Nicholson conjecture holds for all primes $p$ less than $p_{81}^{*}$, except $p \in\{2,3,5,7\}$. Since we do not explicitly know $p_{81}^{*}$, (though an exhaustive search has now verified that $p_{81}^{*}>2^{64}$ [29], see also the discussion in [28]), a safe fully explicit statement is that both the Firoozbakht and Nicholson conjectures are verified for all primes $p<2^{64} \approx 1.844 \times 10^{19}$. Note that a brief computation [44] (less than $4 \mathrm{~min}$ ) yields $\pi\left(2^{64}\right)=425,656,284,035,217,743 \approx 425 \times 10^{15}$.

\section{Sufficient Conditions for the Farhadian Conjecture}

The Farhadian conjecture is a little trickier to deal with. Again using the fact that $e^{x}-1>x$ we can deduce a sufficient condition.

Sufficient condition 3. (Farhadian)

$$
g_{n}<\frac{p_{n} \ln \left(p_{n} \frac{\ln n}{\ln p_{n}}\right)}{n}=\frac{p_{n}\left(\ln p_{n}+\ln \ln n-\ln \ln p_{n}\right)}{n} ; \quad(n>4) .
$$

Now inside the brackets use the lower bound $p_{n} \geq n \ln n$ (valid for $n \geq 1$ ), and the upper bound $p_{n} \leq n \ln (n \ln n)$ (valid for $n \geq 6$ ). This gives a new slightly stronger sufficient condition.

\section{Sufficient condition 4. (Farhadian)}

$$
g_{n}<\frac{p_{n}(\ln (n \ln n)+\ln \ln n-\ln \ln (n \ln (n \ln n)))}{n} ; \quad(n>6) .
$$

Now use Dusart's result [14] that for $n \geq 2$ we have $p_{n}>n(\ln (n \ln n)-1)$ to deduce another yet even slightly stronger sufficient condition.

\section{Sufficient condition 5. (Farhadian)}

$$
g_{n}<f(n)=(\ln (n \ln n)-1)(\ln (n \ln n)+\ln \ln n-\ln \ln (n \ln (n \ln n))) ; \quad(n>6) .
$$

It is now a somewhat tedious but entirely straightforward exercise in elementary calculus to verify that this function $f(n)$ is indeed monotone increasing as a function of $n$. A posteriori, we shall verify that this last sufficient condition is strong enough to be useful, and weak enough to be true over the domain of interest. 


\section{Verifying the Farhadian Conjecture for All Primes $p<2^{64}$}

The logic is the same as for the Firoozbakht and Nicholson conjectures. If the sufficient condition for the Farhadian conjecture holds at the beginning of the interval $p_{n} \in\left[p_{i}^{*}, p_{i+1}^{*}-1\right]$, then it certainly holds on the entire interval. Explicitly checking a table of maximal prime gaps [32-40], we inspect the ratios

$$
\frac{g_{i}^{*}}{\left(\ln \left(n_{i}^{*} \ln n_{i}^{*}\right)-1\right)\left(\ln \left(n_{i}^{*} \ln n_{i}^{*}\right)+\ln \ln n_{i}^{*}-\ln \ln \left(n_{i}^{*} \ln \left(n_{i}^{*} \ln n_{i}^{*}\right)\right)\right)}
$$

and verify that they are less than unity for $i \in[5,80]$. Consequently the Farhadian conjecture certainly holds on the interval $\left[p_{5}^{*}, p_{81}^{*}-1\right]$, from $p_{5}^{*}=89$ up to just before the beginning of the 81 st maximal prime gap, $p_{81}^{*}-1$, even if we do not yet know the value of $p_{81}^{*}$. Then explicitly checking the primes below $p_{5}^{*}=89$ the Farhadian conjecture is verified to hold for all primes $p$ less than $p_{81}$, except $p \in\{2,3,5,7\}$. Since we do not explicitly know $p_{81}^{*}$, (though an exhaustive search has now verified that $p_{81}^{*}>2^{64}$ [29], see also the discussion in [28]), a safe fully explicit statement is that the Farhadian conjecture is verified for all primes $p<2^{64} \approx 1.844 \times 10^{19}$.

\section{Discussion}

While Kourbatov's recent work [26-28] yields a useful and explicit domain of validity for the Firoozbakht conjecture, (ultimately, see [28,29], for all primes $p<2{ }^{64}$ ), the present article first slightly extends this domain of validity (to all primes up to the as yet unknown location of the 81st maximal prime gap: $p<p_{81}^{*}$ ), and second and more significantly obtains identical domains of validity for the related but somewhat stronger Nicholson and Farhadian conjectures. The analysis has been presented in such a way that it can now be semi-automated. Upon discovery, every new maximal prime gap $g_{i}^{*}$ can, (as long as one can also calculate the corresponding $n_{i}^{*}=\pi\left(p_{i}^{*}\right)$, see for instance [44-47]), be used to push the domain of validity a little further.

Some cautionary comments are in order: verification of these conjectures up to some maximal prime, however large, does not guarantee validity for all primes. Note that by the prime number theorem $\pi(n) \sim \operatorname{li}(n)$ so

$$
\frac{\ln \left(p_{n}\right)}{n}=\frac{\ln \left(p_{n}\right)}{\pi\left(p_{n}\right)} \sim \frac{\ln \left(p_{n}\right)}{\operatorname{li}\left(p_{n}\right)} .
$$

Now certainly $\ln (p) / \operatorname{li}(p)$ is monotone decreasing, which is good. On the other hand $\pi(x)-\operatorname{li}(x)$ changes sign infinitely often, (this is the Skewes phenomenon [48-51]), so that the monotone decreasing function $\ln \left(p_{n}\right) / \operatorname{li}\left(p_{n}\right)$ both over-estimates and under-estimates the quantity of interest $\ln \left(p_{n}\right) / n$, which is not so good. Now this observation does not disprove the Firoozbakht conjecture, but it does indicate where there might be some potential difficulty.

On a more positive note, the Firoozbakht conjecture most certainly must hold when averaged over suitably long intervals. It is an elementary consequence of the Chebyshev theorems that $p_{m} p_{n}>p_{m+n}$, see [1-3]. But then $p_{n}^{2}>p_{2 n}$, and $p_{n}^{3}>p_{n} p_{2 n}>p_{3 n}$. In general $\left(p_{n}\right)^{m}>p_{n m}$ and so $\ln p_{n}>\ln p_{n m} / m$. Consequently

$$
\frac{\ln \left(p_{n}\right)}{n}>\frac{\ln \left(p_{n m}\right)}{n m} \text {. }
$$

This is much weaker than the usual Firoozbakht conjecture, but enjoys the merit of being unassailably true.

Funding: This research was supported by the Marsden Fund, administered by the Royal Society of New Zealand.

Acknowledgments: I particularly wish to thank both Alexei Kourbatov and John Nicholson for their interest and useful comments.

Conflicts of Interest: The author declares no conflict of interest. 


\section{References}

1. Ribenboim, P. The Little Book of Big Primes; Springer: New York, NY, USA, 1991.

2. Ribenboim, P. The New Book of Prime Number Records; Springer: New York, NY, USA, 1996.

3. Ribenboim, P. The Little Book of Bigger Primes; Springer: New York, NY, USA, 2004.

4. Wells, D. Prime Numbers: The Most Mysterious Figures in Math; John Wiley: Hoboken, NJ, USA, 2005.

5. Cramér, H. Some theorems concerning prime numbers. Ark. Mat. Astron. Phys. 1920, 15, 5.

6. Cramér, H. On the order of magnitude of the difference between consecutive prime numbers. Acta Arith. 1936, 2, 23-46. [CrossRef]

7. Goldston, D.A. On a result of Littlewood concerning prime numbers. Acta Arith. 1982, 3, 263-271. [CrossRef]

8. Rosser, J.B. The $n^{\prime}$ th Prime is Greater than $n \ln n$. Proc. London Math. Soc. 1938, 45, 21-44.

9. Rosser, J.B. Explicit Bounds for some functions of prime numbers. Am. J. Math. 1941, 63, 211-232. [CrossRef]

10. Cesàro, E. Sur une formule empirique de M. Pervouchine. Comptes Rendus 1894, 119, 848-849.

11. Cipolla, M. La determinazione assintotica dell' $n^{i m o}$ numero primo. Mat. Napoli 1902, 3, 132-166.

12. Rosser, J.B.; Schoenfeld, L. Approximate Formulas for Some Functions of Prime Numbers. Ill. J. Math. 1962, 6, 64-97. [CrossRef]

13. Sándor, J. On certain sequences and series with applications in prime number theory. Gaz. Mat. Met. Inf. 1985, 6, 1-2.

14. Dusart, P. The $k^{\text {th }}$ prime is greater than $k(\ln k+\ln \ln k-1)$ for $k \geq 2$. Math. Comput. 1999, 68, 411-415. [CrossRef]

15. Lowry-Duda, D. A Short Note on Gaps between Powers of Consecutive Primes. arXiv 2017, arXiv:1709.07847.

16. Dusart, P. Estimates of some functions over primes without RH. arXiv 2010, arXiv:1002.0442.

17. Trudgian, T. Updating the error term in the prime number theorem. Ramanujan J. 2016, 39, 225. [CrossRef]

18. Dusart, P. Explicit estimates of some functions over primes. Ramanujan J. 2018, 45, 227. [CrossRef]

19. Axler, C. New estimates for some functions defined over primes. arXiv 2017, arXiv:1703.08032.

20. Visser, M. Primes and the Lambert $W$ function. Mathematics 2018, 6, 56. [CrossRef]

21. Andrica, D. Note on a conjecture in prime number theory. Studia Univ. Babes-Bolyai Math. 1986, 31, 44-48, ISSN 0252-1938.

22. Visser, M. Variants on Andrica's conjecture with and without the Riemann hypothesis. Mathematics 2018, 6, 289. [CrossRef]

23. Visser, M. Strong version of Andrica's conjecture. arXiv 2018, arXiv:1812.02762.

24. Farideh Firoozbakht. Unpublished. 1982. Available online: https:/ /www.primepuzzles.net/thepuzzlers/ Firoozbakht.htm (accessed on 30 July 2019).

25. Rivera, C. (Ed.) Conjecture 30. The Firoozbakht Conjecture. 2002. Available online: https://www. primepuzzles.net/conjectures/conj_030.htm (accessed on 30 July 2019).

26. Kourbatov, A. Verification of the Firoozbakht conjecture for primes up to four quintillion. Int. Math. Forum 2015, 10, 283-288. [CrossRef]

27. Kourbatov, A. Upper bounds for prime gaps related to Firoozbakht's conjecture. J. Integer Seq. 2015, 18, 15.11.2.

28. Kourbatov, A. Prime Gaps: Firoozbakht Conjecture. Updated March 2019. Available online: http:/ / www. javascripter.net/math/primes / firoozbakhtconjecture.htm (accessed on 30 July 2019).

29. Nicely, T.R. First Occurrence Prime Gaps. Updated March 2019. Available online: http:/ /www.trnicely.net/ gaps/gaplist.html (accessed on 30 July 2019).

30. Nicely, T.R.; Nyman, B. New prime gaps between $10^{15}$ and $5 \times 10^{16}$. J. Integer Seq. 2003, 6, 03.3.1.

31. Oliveira e Silva, T.; Herzog, S.; Pardi, S. Empirical verification of the even Goldbach conjecture and computation of prime gaps up to $4 \times 10^{18}$. Math. Comp. 2014, 83, 2033-2060. [CrossRef]

32. For All of the Maximal Prime Gaps up to $\left(80, g_{80}^{*}, n_{80}^{*}, p_{80}^{*}\right)$. Available online: https://en.wikipedia.org/ wiki/Prime_gap (accessed on 30 July 2019).

33. For All of the Maximal Prime Gaps up to $\left(80, g_{80}^{*}, n_{80}^{*}, p_{80}^{*}\right)$. Available online: http://trnicely.net/\#Maximal (accessed on 30 July 2019).

34. For All of the Maximal Prime Gaps up to $\left(75, g_{75}^{*}, p_{75}^{*}\right)$. Available online: http://primerecords.dk/ primegaps / maximal.htm (accessed on 30 July 2019). 
35. For All of the Maximal Prime Gaps up to $\left(75, g_{75}^{*}, p_{75}^{*}\right)$. Available online: https:/ / primes.utm.edu/notes/ GapsTable.html (accessed on 30 July 2019).

36. Sloane, N.J.A. Sequence A005250 in The On-Line Encyclopedia of Integer Sequences. Available online: https: / / oeis.org/A005250 (accessed on 30 July 2019).

37. Sloane, N.J.A. Sequence A002386 in The On-Line Encyclopedia of Integer Sequences. Available online: https: / / oeis.org/A002386 (accessed on 30 July 2019).

38. Sloane, N.J.A. Sequence A005669 in The On-Line Encyclopedia of Integer Sequences. Available online: https: / / oeis.org/A005669 (accessed on 30 July 2019).

39. Sloane, N.J.A. Sequence A000101 in The On-Line Encyclopedia of Integer Sequences. Available online: https: / / oeis.org/A000101 (accessed on 30 July 2019).

40. Sloane, N.J.A. Sequence A107578 in The On-Line Encyclopedia of Integer Sequences. Available online: https:/ / oeis.org/A107578 (accessed on 30 July 2019).

41. Nicholson, J. See Sloane, N.J.A. Sequence A182514 in The On-Line Encyclopedia of Integer Sequences. Unpublished. 2013. Available online: https:/ / oeis.org/A182514 (accessed on 30 July 2019).

42. Farhadian, R. A New Conjecture on the Primes. Available online: https://www.primepuzzles.net/ conjectures/Reza\%20Faradian\%20Conjecture.pdf (accessed on 30 July 2019).

43. Farhadian, R.; Jakimczuk, R. On a new conjecture of prime numbers. Int. Math. Forum 2017, 12, 559-564. [CrossRef]

44. Walisch, K. Primecount: A Program for Computing $\pi(x)$. Available online: https://github.com/kimwalisch/ primecount (accessed on 30 July 2019).

45. Deleglise, M.; Rivat, J. Computing $\pi(x)$ : The Meissel, Lehmer, Lagarias, Miller, Odlyzko method. Math. Comp. 1996, 65, 235-245. [CrossRef]

46. Lagarias, J,C.; Miller, V.S.; Odlyzko, A.M. Computing $\pi(x)$ : The Meissel-Lehmer Method. Math. Comput. 1985, 44, 537-560. [CrossRef]

47. Oliveira e Silva, T. Computing $\pi(x)$ : The combinatorial method. Rev. DETUA 2006, 4, 759-768.

48. Skewes, S. On the Difference $\pi(x)-\operatorname{li}(x)$. J. Lond. Math. Soc. 1933, 8, 277-283. [CrossRef]

49. Skewes, S. On the Difference $\pi(x)-\operatorname{li}(x)$. II. Proc. Lond. Math. Soc. 1955, 5, 48-70. [CrossRef]

50. Lehman, R. S. On the Difference $\pi(x)-\operatorname{li}(x)$. Acta Arith. 1966, 11, 397-410. [CrossRef]

51. Te Riele, H.J.J. On the Sign of the Difference $\pi(x)-\operatorname{li}(x)$. Math. Comput. 1987, 48, 323-328. [CrossRef]

(C) 2019 by the author. Licensee MDPI, Basel, Switzerland. This article is an open access article distributed under the terms and conditions of the Creative Commons Attribution (CC BY) license (http://creativecommons.org/licenses/by/4.0/). 